OPEN ACCESS

Edited by:

Barbara Altieri,

University Hospital of Wuerzburg,

Germany

Reviewed by:

Peter lgaz,

Semmelweis University, Hungary

Jaydira Del Rivero,

National Cancer Institute (NIH),

United States

${ }^{*}$ Correspondence:

Alfredo Berruti

alfredo.berruti@gmail.com

${ }^{t}$ These authors have contributed equally to this work and share first authorship

Specialty section:

This article was submitted to Cancer Endocrinology, a section of the journal

Frontiers in Endocrinology

Received: 28 February 2021 Accepted: 31 March 2021

Published: 22 April 2021

Citation:

Cosentini D, Turla A, Carminati O

Grisanti S, Ferrari VD, Laganà $M$

Rosti G, Sigala S and Berruti A (2021) Case Report: Exceptional Response to

Second Line Temozolomide Therapy in a Patient With Metastatic Adrenocortical Carcinoma.

Front. Endocrinol. 12:674039. doi: 10.3389/fendo.2021.674039

\section{Case Report: Exceptional Response to Second Line Temozolomide Therapy in a Patient With Metastatic Adrenocortical Carcinoma}

\author{
Deborah Cosentini ${ }^{1 \dagger}$, Antonella Turla ${ }^{1 \dagger}$, Ornella Carminati ${ }^{2}$, Salvatore Grisanti ${ }^{1}$, \\ Vittorio Domenico Ferrari ${ }^{1}$, Marta Laganà ${ }^{1}$, Giovanni Rosti ${ }^{3}$, Sandra Sigala ${ }^{4}$ \\ and Alfredo Berruti ${ }^{\text {1* }}$
}

\begin{abstract}
${ }^{1}$ Medical Oncology Unit, Azienda Socio Sanitaria Territoriale (ASST) Spedali Civili, Department of Medical and Surgical Specialties, Radiological Sciences, and Public Health, University of Brescia, Brescia, Italy, ${ }^{2}$ Medical Oncology, Department of Oncology and Hematology, Azienda Unità Sanitaria Locale (AUSL), Romagna, Ravenna, Italy, ${ }^{3}$ Medical Oncology, Fondazione IRCCS Policlinico San Matteo, Pavia, Italy, ${ }^{4}$ Section of Pharmacology, Department of Molecular and Translational Medicine, University of Brescia, Brescia, Italy
\end{abstract}

Background: In a recently published retrospective case series, Temozolomide was found active as second line approach in advanced ACC patients. The disease control rate obtained, however, was short-lived. We report here an ACC patient with extensive metastatic disease who obtained a remarkable long lasting response with this alkylating agent.

Case Presentation: a 22-year-old female patient with ACC presented at our Medical Oncology Department in poor general condition due the presence of extensive metastatic pulmonary involvement. The disease had progressed to etoposide, doxorubicin and cisplatin plus mitotane therapy. Second line temozolomide therapy was prescribed leading to a progressive improvement of patient general conditions. The disease restaging after 12 cycles revealed a complete response of lung lesions and the patient was free from progression for $14+$ months.

Conclusion: Temozolomide therapy could be exceptionally efficacious in the management of ACC patients. The molecular mechanisms of sensitivity and resistance to this drug should be carefully studied, in order to select the patients destined to obtain a significant clinical benefit to the drug.

Keywords: adrenal tumor, alkylating drug, progesterone, treatment, ACC

\section{BACKGROUND}

Adrenocortical carcinoma (ACC) is a rare and aggressive tumor with an incidence of 0.7-2 new cases per million populations per year (1). Surgery is the mainstay of therapy for localized diseases and patients with moderate to high risk of disease relapse and death are addressed to adjuvant mitotane therapy (2). The standard systemic treatment for advanced/metastatic tumors, not 
amenable to surgical resection, is the combination of etoposide, doxorubicin and cisplatin plus mitotane (EDP-M) (3). The results of a large multicenter randomized clinical trial have demonstrated that the efficacy of this regimen is limited with a disease response of about $25 \%$ and a median overall survival of 14 months (4). However, a single Institution case series, in which surgery of residual disease after EDP-M was systematically performed when feasible, revealed that few patients (7\%) can obtain a pathological complete response and a long term disease control (5). These data suggest that EDP-M scheme can be sometimes very efficacious. Few options are available for patients with disease progression to EDP-M. Gemcitabine plus metronomic capecitabine is a second line approach recommended by international guidelines (1), but the efficacy of this regimen is modest (6). Several target therapies substantially failed to demonstrate activity in ACC $(7,8)$ and the role of immunotherapy is currently under investigation (9).

In a recently conducted retrospective study, single agent temozolomide was found active as second line approach in advanced ACC patients with a response rate observed in $20 \%$ of patients. The disease control rate obtained, however, was short-lived (10).

Here, we presented the case of a 22 -year-old female patient with advanced ACC reporting a dramatic disease response to temozolomide therapy.

\section{CASE PRESENTATION}

A 22-year-old woman from Sicily presented in August 2019 at the Medical Oncology Unit of Santa Maria delle Croci hospital in Ravenna, Italy, with severe hypertension, hirsutism, oligomenorrhea, and epigastric pain. A CT scan showed a huge left adrenal mass (100x110mm) and multiple lung metastasis. Hormonal assessment revealed elevated plasma and urinary cortisol levels and elevated androgen levels. The patient's general conditions were compromised due to a severe Cushing's syndrome. A tumor biopsy was performed confirming the ACC diagnosis. The patient was immediately addressed to first line chemotherapy with the EDP-M regimen, administered in association with metyrapone, to rapidly obtain a control of hormone hypersecretion (11). A minimal disease response of lung metastases and no change of the primary tumor lesion was obtained after 2 chemotherapy cycles. The patient general conditions, however, consistently improved. Antineoplastic therapy was continued and, from the $3^{\text {rd }}$ cycles onwards, mitotane serum levels had attained the therapeutic interval, ranging between 14.5 and $20.1 \mathrm{ng} / \mathrm{ml}$. Unfortunately, a huge disease progression was observed at TC restaging after 5 cycles.

The patient was then referred to the Medical Oncology Unit in Brescia in January 2020. On admission her general conditions were poor (ECOG performance status 3). She suffered from uncontrolled pain, nausea, asthenia and dyspnea. Oxygen saturation was low (SpO2 88\%), so continuous oxygen therapy was instituted.

The CT scan confirmed the voluminous left adrenal mass (160x110 mm in size) infiltrating the left kidney and revealed a dramatic disease progression in lung with multiple lesions affecting both hemithoraxes (Figure 1).

Due her serious conditions we deemed the patient not eligible to further intravenous chemotherapy. She was then discharged and entrusted to home palliative care in Sicily with the prescription of oral temozolomide at the dose of $200 \mathrm{mg} / \mathrm{m} 2 /$ die given for 5 consecutive days every 28 days and daily. Megestrol acetate $160 \mathrm{mg}$ every day was also concomitantly prescribed to support appetite and patient kenesthesis. Mitotane was continued.

Progressively, the patient general conditions improved with a normalization of oxygen saturation and pain control. Temozolomide therapy was well tolerated without any relevant toxicity. After 2 months her quality of life returned to normal and megestrol acetate was interrupted while continuing both temozolomide and mitotane therapies. The CT restaging performed in March 2020 showed a partial response of lung metastases with a minimal response of the adrenal mass. The extent of lung disease further decreased at a subsequent CT in June 2020. In September the patient returned to Brescia for a follow-up visit. A CT scan revealed a complete disease response of lung metastases and a partial response of the abdominal lesion

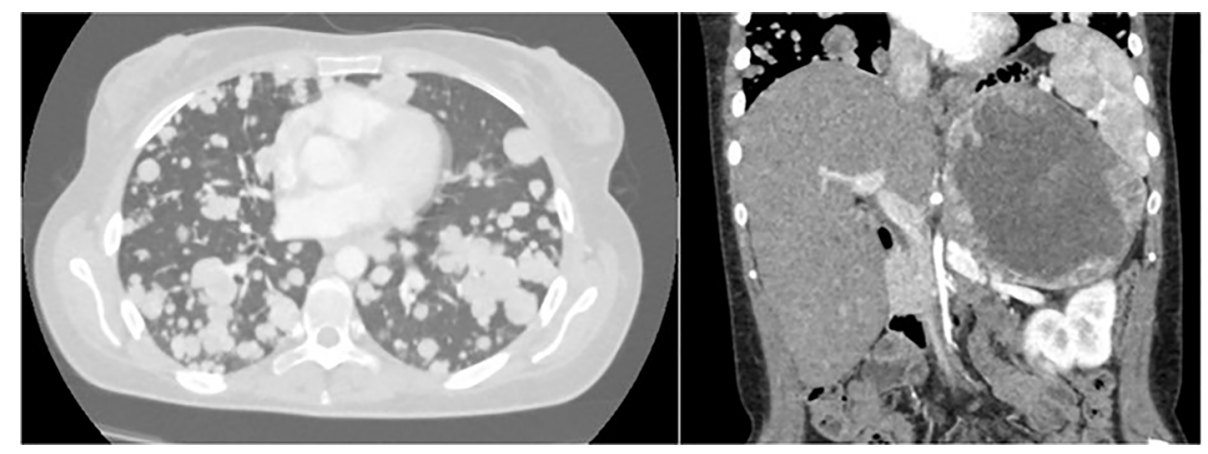

FIGURE 1 | Multiple pulmonary metastases and adrenal mass detected by CT Scan (January 2020). 
with dimension reduction $(60 \mathrm{~mm})$ and necrosis increase (Figure 2). The FDG PET scan confirmed the complete response of the lung lesions and, as regard the abdominal mass, a small peripheral uptake was described with an extensive central area with no FDG (fluorodesoxyglucose) uptake (necrosis) (Figure 2).

The last CT scan (December 2020) revealed a stable disease with respect to the previous control in September. At the last follow-up examination, on February $25^{\text {th }}$, the patient general conditions were excellent (ECOG performance status 0 ). She was still on Temozolomide treatment (12 total cycles) along with mitotane and was free from progression for 14 months.

A Next Generation Sequencing (NGS) analysis, with a wide panel of genes (genic variant in hotspot regions of 35 genes -AKT1, ALK, AR, BRAF, CDK4, CTNNB1, DDR2, EGFR, ERBB2, ERBB3, ERBB4, ESR1, FGFR2, FGFR3, GNA11, GNAQ, HRAS, IDH1,
IDH2, JAK1, JAK2, JAK3, KIT, KRAS, MAP2K1, MAP2K2, MET, MTOR, NRAS, PDGFRA, PIK3CA, RAF1, RET, ROS1, SMO; amplification of 19 genes- ALK, AR, BRAF, CCND1, CDK4, CDK6, EGFR, ERBB2, FGFR1, FGFR2, FGFR3, FGFR4, KIT, KRAS, MET, MYC, MYCN, PDGFRA, PIK3CA; rearrangements of 23 genes- ABL1, AKT3, ALK, AXL, BRAF, ERG, ETV1, ETV4, ETV5, EGFR, ERBB2, FGFR1, FGFR2, FGFR3, MET, NTRK1, NTRK2, NTRK3, FDGFRA, PPARG, RAF1, RET, ROS1) was performed on biopsy tumor samples at diagnosis. A genic variant in CTNN1B exon 3 (p.Met14Leu) was found (Table 1). This variant has never been published as pathogenetic in literature and it is not present in ClinVar, whereas it is categorized as VUS (variance of uncertain significance) in the VARSOME database. O6methylguanine-DNA methyl-transferase (MGMT) and progesterone receptor (PgR) expression could not be assessed, due to insufficient tumor materials.

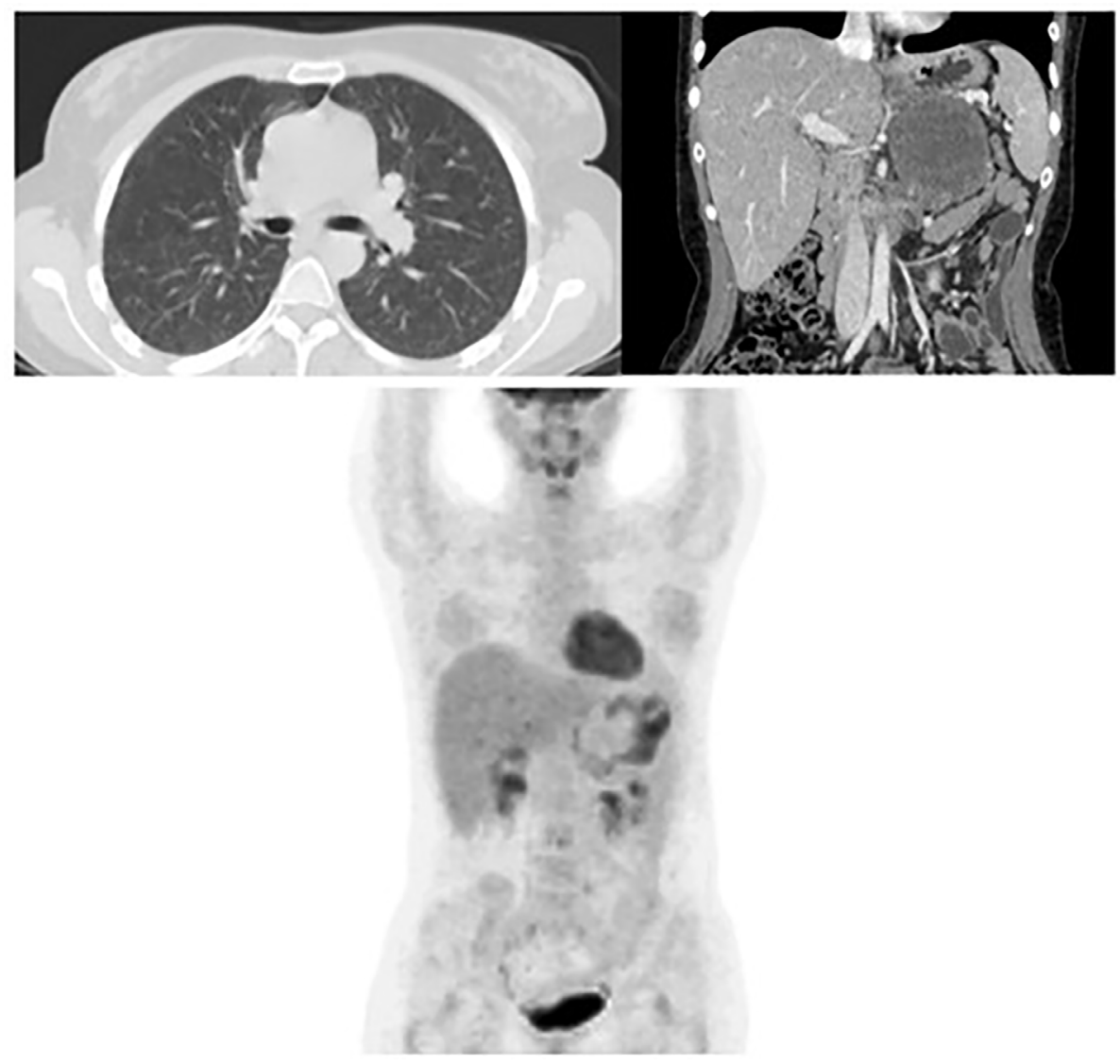

FIGURE 2 | Complete response of the bilateral lung metastases and reduction of the primary adrenal lesion at the CT Scan after 8 cycles of temozolomide. FDG PET confirming the complete response of lung lesions and a small peripheral area of FDG uptake at the residual adrenal mass. (September 2020).

TABLE 1 | Genic variant detected by NGS.

\begin{tabular}{llllllll}
\hline Chromosome & Gene & Genic region & Proteic variant (cDNA) & Variant type & Coverage & Allelic frequency & Reference sequence \\
\hline 3 & CTNN1B & Exon 3 & p.Met14Leu (c.40A>C) & Nucleotide sustitution & 1759 & $39.9 \%$ & NM_001098210.1 (GenBank)
\end{tabular}




\section{DISCUSSION}

Chemotherapy + mitotane (EDP-M) is modestly active as first first-line approach in the management of patients with advanced/metastatic ACC. At disease progression to EDP-M, the further administration of cytotoxic drugs gave disappointing results. At the best of our knowledge, this is the first ACC case that has obtained a dramatic response with a second-line chemotherapy. This result is even more relevant if we consider that the patient in question had an extensive disease burden and her performance status was very low. The latter condition is known to be an independent factor of poor efficacy of systemic antineoplastic treatments in general and ACC in particular (1).

It is certainly not clear why second-line temozolomide was particularly effective in this case. The drug was found to be able to induce a significant cytotoxic effect in ACC cells in vitro (12). However, in a recent Italian case series of pre-treated ACC patients, it proved to be moderately active with a disease response rate observed in $20 \%$ of treated cases, but poorly effective, as disease control was short lived (3.5 months on average) (10).

The addition of megestrol acetate for palliation may have contributed in the first weeks to support the patient's performance status and favored the cytotoxic effect of temozolomide. A recent paper by our group has in fact demonstrated an antineoplastic effect of progesterone in ACC cell lines and primary ACC cultures (13). Unfortunately, due to insufficient tissue material, it was not possible to test the expression of the PgR and MGMT in the primary tumor, which are known predictors of efficacy of megestrol acetate and temozolomide, respectively. The NGS performed failed to provide a potentially valuable predictive parameter.

In conclusion, the exceptional and long lasting disease response obtained with temozolomide in this ACC patient, suggests that this drug can be very efficacious in this setting. Temozolomide deserve to be further tested in ACC patients with

\section{REFERENCES}

1. Fassnacht M, Assie G, Baudin E, Eisenhofer G, de la Fouchardiere C, Haak HR, et al. Adrenocortical Carcinomas and Malignant Phaeochromocytomas: ESMO-EURACAN Clinical Practice Guidelines for Diagnosis, Treatment and Follow-Up. Ann Oncol (2020) 31(11):1476-90. doi: 10.1016/ j.annonc.2020.08.2099

2. Berruti A, Grisanti S, Pulzer A, Claps M, Daffara F, Loli P, et al. Long-Term Outcomes of Adjuvant Mitotane Therapy in Patients With Radically Resected Adrenocortical Carcinoma. J Clin Endocrinol Metab (2017) 102(4):1358-65. doi: 10.1210/jc.2016-2894

3. Berruti A, Terzolo M, Sperone P, Pia A, Della Casa S, Gross DJ, et al. Etoposide, Doxorubicin and Cisplatin Plus Mitotane in the Treatment of Advanced Adrenocortical Carcinoma: A Large Prospective Phase II Trial. Endocr Relat Cancer (2005) 12(3):657-66. doi: 10.1677/erc.1.01025

4. Fassnacht M, Terzolo M, Allolio B, Baudin E, Haak H, Berruti A, et al. Combination Chemotherapy in Advanced Adrenocortical Carcinoma. N Engl J Med (2012) 366(23):2189-97. doi: 10.1056/NEJMoa1200966

5. Laganà $M$, Grisanti S, Cosentini D, Ferrari VD, Lazzari B, Ambrosini R, et al. Efficacy of the EDP-M Scheme Plus Adjunctive Surgery in the Management of Patients With Advanced Adrenocortical Carcinoma: The Brescia Experience. Cancers (Basel) (2020) 12(4):941. doi: 10.3390/cancers12040941 the aim of identifying predictive factors of efficacy in order to select the patients destined to obtain a significant clinical benefit to the drug.

\section{DATA AVAILABILITY STATEMENT}

The raw data supporting the conclusions of this article will be made available by the authors, without undue reservation.

\section{ETHICS STATEMENT}

The studies involving human participants were reviewed and approved by Brescia ethics committee. The patients/participants provided their written informed consent to participate in this study. Written informed consent was obtained from the individual(s) for the publication of any potentially identifiable images or data included in this article.

\section{AUTHOR CONTRIBUTIONS}

$\mathrm{AB}$ conceived the idea of this manuscript. $\mathrm{OC}, \mathrm{VF}, \mathrm{GR}$, and $\mathrm{AB}$ clinically followed the patient. DC, AT, and ML collected and interpreted the patient clinical data and wrote the manuscript. All authors contributed to the article and approved the submitted version.

\section{FUNDING}

This study was supported in part by FIRM onlus, Cremona, Italy and by Associazione Italiana per la Ricerca contro il Cancro (AIRC), IG: 14411.

6. Sperone P, Ferrero A, Daffara F, Priola A, Zaggia B, Volante M, et al. Gemcitabine Plus Metronomic 5-Fluorouracil or Capecitabine as a Second-/Third-Line Chemotherapy in Advanced Adrenocortical Carcinoma: A Multicenter Phase II Study. Endocr Relat Cancer (2010) 17 (2):445-53. doi: 10.1677/ERC-09-0281

7. Terzolo M, Daffara F, Ardito A, Zaggia B, Basile V, Ferrari L, et al. Management of Adrenal Cancer: A 2013 Update. J Endocrinol Invest (2014) 37(3):207-17. doi: 10.1007/s40618-013-0049-2

8. Grisanti S, Cosentini D, Laganà M, Abate A, Rossini E, Sigala S, et al. Are We Failing in Treatment of Adrenocortical Carcinoma? Lights and Shadows of Molecular Signatures. Curr Opin Endocr Metab Res (2019) 8:80-7. doi: 10.1016/j.coemr.2019.07.007

9. Grisanti S, Cosentini D, Laganà M, Volta $\mathrm{AD}$, Palumbo C, Massimo Tiberio GA, et al. The Long and Winding Road to Effective Immunotherapy in Patients With Adrenocortical Carcinoma. Future Oncol (2020) 16(36):301720. doi: $10.2217 /$ fon-2020-0686

10. Cosentini D, Badalamenti G, Grisanti S, Basile V, Rapa I, Cerri S, et al. Activity and Safety of Temozolomide in Advanced Adrenocortical Carcinoma Patients. Eur J Endocrinol (2019) 181(6):681-9. doi: 10.1530/ EJE-19-0570

11. Claps M, Cerri S, Grisanti S, Lazzari B, Ferrari V, Roca E, et al. Adding Metyrapone to Chemotherapy Plus Mitotane for Cushing's Syndrome Due to 
Advanced Adrenocortical Carcinoma. Endocrine (2018) 61(1):169-72. doi: $10.1007 / \mathrm{s} 12020-017-1428-9$

12. Creemers SG, van Koetsveld PM, van den Dungen ES, Korpershoek E, van Kemenade FJ, Franssen GJ, et al. Inhibition of Human Adrenocortical Cancer Cell Growth by Temozolomide in Vitro and the Role of the MGMT Gene. J Clin Endocrinol Metab (2016) 101(12):4574-84. doi: 10.1210/ jc.2016-2768

13. Fragni M, Fiorentini C, Rossini E, Fisogni S, Vezzoli S, Bonini SA, et al. In Vitro Antitumor Activity of Progesterone in Human Adrenocortical Carcinoma. Endocrine (2019) 63(3):592-601. doi: 10.1007/s12020-018$1795-\mathrm{x}$
Conflict of Interest: The authors declare that the research was conducted in the absence of any commercial or financial relationships that could be construed as a potential conflict of interest.

Copyright (c) 2021 Cosentini, Turla, Carminati, Grisanti, Ferrari, Laganà, Rosti, Sigala and Berruti. This is an open-access article distributed under the terms of the Creative Commons Attribution License (CC BY). The use, distribution or reproduction in other forums is permitted, provided the original author(s) and the copyright owner(s) are credited and that the original publication in this journal is cited, in accordance with accepted academic practice. No use, distribution or reproduction is permitted which does not comply with these terms. 